\title{
Editorial
}

\section{Letting go of the old and embracing the new}

Change ... is not a one-step process, for academics, but one that sees them in transition, as they let go of the old and prepare to embrace the new. A key question that needs to be asked is: How much of the past do they need to let go in order to embrace the future? (McMurray, 2001: 80)

A major focus of the research and practice in the field of Learning Technology is implementing and developing learning technologies in order to change or influence the student learning experience. We are all aware, however, that the process of developing or implementing learning technologies often requires adaptation or changes in staff roles and skills, in organizational culture and structures, in strategies and policies, in attitudes and perceptions as in well as the theories and models we use to explain and understand the learning and teaching experience. In this issue of $A L T-J$ we present ten papers. On first reading these papers represent quite an eclectic mixture of subjects and methods: a closer inspection, however, reveals a common theme that runs through all the papers, that of change. Linked to this theme are notions of evolution, innovation and experimentation.

The first two papers present a commentary on a paper published in a previous edition of $A L T-J$. The following six papers were presented at the ALT-C annual conference entitled 'Changing Learning Environments' in Edinburgh last year, while the final two papers report on the results of two different evaluation studies.

The papers by Conole et al., by Davis and Denning, and by Wise represent an attempt to develop the relationship between $A L T-J$ authors and readers and facilitate a more interactive and perhaps reflective dialogue between the two parties. Conole, Oliver and Seale summarize readers' comments on an article by Davis and Denning published in issue 9.2 of this journal entitled 'Almost as helpful as good theory: some conceptual possibilities for the online classroom'. This is followed by a response from Davis and Denning who 
remark that 'some of the comments made by readers have indicated a way forward'. Wise describes a program he has written using Javascript that is designed to help life sciences students prepare a research protocol. In an attempt to engage with $A L T-J$ readers, he has made the program available on the Web and invites comments and suggestions from $A L T-J$ readers for 'future collaborative research'.

The notion of change through meaningful dialogue is also highlighted in the papers by McAvinia and by Lockyer et al. McAvinia describes two approaches to the design and delivery of Web-based key skills resources: the central resource and customized, individualized sets of departmental pages. McAvinia argues that the adaptation of the centralized key skills resources to departmental contexts is not simply a question of changing the packaging or 're-branding' them. It requires a meaningful dialogue with members of the department in order to produce a product that has contextual relevance for students. Lockyer, Patterson, Rowland and Hearne describe a small pilot study, which used asynchronous Web-based communication tools with student teachers in Australia to facilitate mentoring and peer support during teaching placements. The study found that while students seemed comfortable using the online peer support, they were not as 'reflective' as the authors had hoped they would be. In considering the implications of their findings Lockyer et al. conclude that changing students' descriptions of teaching experience into reflections on teaching experience may be achieved by 'preparing both the students and the lecturers to identify the difference between a reflective and descriptive posting and developing strategies to support reflection'.

The papers by Riley and Gunn et al. use knowledge gained from theory and practice in order to develop our understanding or use of learning technology. The paper by Gunn, French, Macleod, McSporran and Conole combines literature review, case studies and feedback from discussion groups held at AlT-C to offer a range of interpretations for the underlying causes and implications of gender differences in computer-supported learning. The paper seeks to change and move our understanding of gender differences beyond considering differences in computer access and skills and challenges us to think about whether it is possible to change the computer-supported learning environments available to students so that they offer more 'widely applicable solutions' to the gender issues identified. The paper by Riley outlines the concept and theoretical underpinnings of 'virtual culture' and explores how it might be developed to evaluate the 'innovative' potential of learning technologies in tertiary education. The notion of 'virtual culture' was first conceived to explain human cultural evolution, but Riley develops and applies the concept to learning technologies by proposing four criteria by which they can be evaluated for their 'virtual culture potential'.

Foster-Jones and Beazleigh, in their paper, describe two Open University metadata projects in order to illustrate how they have tried to apply the IMS Object metadata scheme. In discussing the implications of their experiences for future work they argue that it is crucial that we invest our efforts and resources in this development phase so that we can contribute to the evolution of metadata standards, using our experiences to create one that best suits our needs'.

Fiddes et al. and Duffy et al. present two experimental studies that attempt to further our knowledge of how student performance in computer-based learning environments 
compares with that in non-computer-based environments. Fiddes, Korabinski, McGuire, Youngson and McMillan present results from an experiment that sets out to compare secondary students' mathematical performance in a computer-based examination with that in a paper-based examination. The experiment was specifically designed to examine the effect of two variables: rewording and medium. A major conclusion drawn from the project is that the assessment medium had no effect on student marks. Duffy, Gilbert, Kennedy and Poon Wai Kwong present a comparison of assessment results achieved by distance learning students and classroom-based students undertaking the same module in a Health Studies degree course. Through a comparison of assessment results the authors found that students studying by distance learning obtained significantly higher end-of-module results than their classroom-based colleagues. The challenge faced by the authors of both papers is to use their results to build on the research of the previous decade in order to develop new ways of interpreting them.

The ten papers in this issue have all addressed in some way issues of change, evolution, innovation or experimentation. Whilst the papers offer us new ways of conceptualizing or understanding our practice they do not encourage us to let go of the old in order to embrace the new. They encourage us to use what we know or have experienced already in order to explore or investigate (with others where appropriate) what might be possible in the future. In answer to the question posed by McMurray, 'How much of the past do they need to let go in order to embrace the future?', the answer is not all of it. Whilst transition does involve a passing from one place to another, the bridge to the future needs a firm foundation in our past experiences.

Jane Seale, Deputy Editor ALT-J Jeff Haywood, ALT-C 2001 Organizing Committee, University of Edinburgh Nora Mogey, ALT-C 2001 Organizing Committee, University of Edinburgh

\section{Reference}

McMurray, D. W. (2001); "The importance of "goodness of fit" between organizational culture and climate in the management of change: a case study in the development of online learning', Association for Learning Technology Journal, 9 (1), 73-83. 